EPJ Web of Conferences 21, 07004 (2012)

DOI: $10.1051 /$ epjconf/20122107004

(C) Owned by the authors, published by EDP Sciences, 2012

\title{
On neutron-induced reaction mechanisms at medium energies
}

\author{
V. Avrigeanu, ${ }^{\text {a }}$, M. Avrigeanu, and F. L. Roman
}

Horia Hulubei National Institute for Physics and Nuclear Engineering, P.O.Box MG-6, BucharestMagurele, Romania

\begin{abstract}
Recent accurate data obtained for the isomeric cross section of the ${ }^{197} \mathrm{Au}(n, 2 n)$ reaction provides an effective opportunity to consider the question of the effective moment of inertia of the nucleus within a consistent model analysis of all available reaction data for the ${ }^{197} \mathrm{Au}$ target nucleus. Moreover, since the corresponding model assumptions are better validated by analysis of the data above $20-30 \mathrm{MeV}$ where only several data with large uncertainties are known, the usefulness of further measurements to be performed at large-scale facilities as SPIRAL-2 and n_TOF, for incident energies up to 40 as well as $100 \mathrm{MeV}$, are underlined.
\end{abstract}

\section{Introduction}

The actual fusion technology programmes requires a well-qualified nuclear database and validated computational tools for reliable neutronics and activation calculations. The generation, maintenance and validation of nuclear data libraries relevant to ITER, IFMIF and DEMO nuclear engineering design demand, among others, improvement of nuclear models and update of databases. Moreover, the neutron energy range concerned in this respect is extended to $50 \mathrm{MeV}$. On the other hand, the cross sections for nuclear reactions induced by fast neutrons below $20 \mathrm{MeV}$ are generally considered to be reasonably well known in spite of many fast neutron reactions for which the several data are either conflicting or incomplete even around $14 \mathrm{MeV}$. It is the reason why having recent sets of accurate measured cross sections still below $20 \mathrm{MeV}$ is highly desirable. Actually the model calculations of these data are most sensitive to the parameters related to nuclei in the early stages of the reaction, i.e. within the pre-equilibrium emission (PE) processes which then become dominating at higher energies. Thus, there is a good opportunity to look for the understanding of the model constraints which are responsible for the calculated cross section variations, concerning particularly (a) the incident energies below $20 \mathrm{MeV}$, where the statistical model (SM) calculations are most sensitive to the parameters related to residual nuclei and emitted particles which are populating them, and (b) the energies above 20-30 MeV, where the PE processes become dominating so that the measured data analysis may better validate the corresponding model assumptions as, e.g., the nuclear potential finite-depth correction in the GDH model for the partial, particle-hole, level density (PLD) formula.

Among the former category of model parameters, especially SM related, some of the most important concern the nuclear level density and and its spin distribution determined by the effective moment of inertia of the nucleus. A particular interest has recently been connected with this issue for nuclei in the transitional region from well deformed to spherical nuclei near the $Z=82$ shell closure (e.g., Refs. [1-3]). Former trials (e.g., [4]) concerned the analysis of the isomeric cross section ratio in order to check the adoption of a variable moment of inertia between the half and $75 \%$ of the rigid-body value $I_{r}$, for the excitation energies from g.s. to the nucleon binding energy, and next to $I_{r}$ around the excitation energy of $15 \mathrm{MeV}$ [5]. Unfortunately it resulted that even the largest related change is still of the same magnitude with the uncertainties associated with the decay schemes and spread of the experimental data, so that a definitive conclusion on this point was precluded. However the new quite

\footnotetext{
a e-mail: vlad.Avrigeanu@nipne.ro
}

This is an Open Access article distributed under the terms of the Creative Commons Attribution-Noncommercial License 3.0, which permits unrestricted use, distribution, and reproduction in any noncommercial medium, provided the original work is properly cited. 
accurate data obtained for the isomeric cross section of the ${ }^{197} \mathrm{Au}(n, 2 n)$ reaction [2] provides a new opportunity which makes the object of the present work.

Moreover, since the corresponding PE model assumptions are better validated by analysis of the data above 20-30 MeV, where only several data with large uncertainties are known, this work underlines the usefulness of further measurements to be performed at large-scale facilities, e.g. SPIRAL-2 [6] and n_TOF [7], for incident energies up to 40 as well as $100 \mathrm{MeV}$. A particular consideration is given to PE parameters and assumptions of the Geometry-Dependent Hybrid (GDH) model - the most important for the medium energies where the global predictions have shown a larger variance with respect to the measured data.

\section{Nuclear models and parameters}

The main purpose of this work has been to avoid the usual question marks associated with the model calculations which combine PE with equilibrium decay of the remaining compound nucleus, in order to enable a stringent test of models for the above-mentioned nuclear processes. Thus, we have analyzed the activation cross sections of ${ }^{197} \mathrm{Au}$ target nucleus using a local parameter set within an updated version of the STAPRE-H95 code [8]. The particular properties of various target nuclei and reaction channels have been considered by using a consistent local parameter set, established on the basis of various independent data in a small range of mass and charge numbers. A generalized GeometryDependent Hybrid (GDH) model $[9,10]$ for PE processes added within STAPRE-H version of the original code [11] includes the angular-momentum conservation [12] and the $\alpha$-particle based on a pre-formation probability $\varphi$ [13] with the values in the present work of 0.25 . The same optical model potential (OMP) and nuclear level density parameters have been used in the framework of the OM, PE and SM models, for calculation of the intra-nuclear transition rates and single-particle level densities at the Fermi level $[10,14,15]$, respectively, in the former case.

The comparison of various calculations, including their sensitivity to model approaches and parameters, has concerned all activation channels for which there are measured data. It has thus avoided the use of model parameters which have been improperly adjusted to take into account properties peculiar to specific nuclei in the decay cascade, considered to be the case for discrepancies observed around the closure of the $h_{11 / 2}$ proton shell.

\subsection{The OMP parameters}

The nucleon optical potential of Koning and Delaroche [16] has obviously been the first option. However, a basic point revealed by these authors just for the slightly deformed ${ }^{197} \mathrm{Au}$ nucleus is that even their spherical local potential does not describe adequately the extrema in the neutron total cross section. Therefore we have applied the SPRT method [17] for validation of the OMP parameters over a wide neutron energy range through analysis of the $s$ - and $p$-wave neutron strength functions, the potential scattering radius $R^{\prime}$ and the energy dependence of the total cross section $\sigma_{T}(E)$ [18]. The recent RIPL-3 recommendations [19] for the low-energy neutron scattering properties and the $\sigma_{T}$ data measured more recently [20] have been used in this respect. The results obtained using the code STAPRE-H and the neutron transmission coefficients provided by using RIPL 1464 potential segment within the code TALYS [21] are shown in Fig. 1 and prove the suitability of the neutron OMP involved in the present work. The same TALYS calculation has been used to obtain the fraction of the neutron reaction cross section corresponding to the collective inelastic scattering cross sections. Typical ratios of the direct inelastic scattering to the total reaction cross sections in the incident energy range from 4 to $40 \mathrm{MeV}$ decrease from $\sim 20$ to $3 \%$, being used for the corresponding decrease of the latter within the rest of reaction cross section calculations.

For calculation of the $\alpha$-particle transmission coefficients we have used the optical potential established previously [22] for emitted $\alpha$-particles, and supported recently by semi-microscopic analysis for $A \sim 90$ nuclei [23]. 


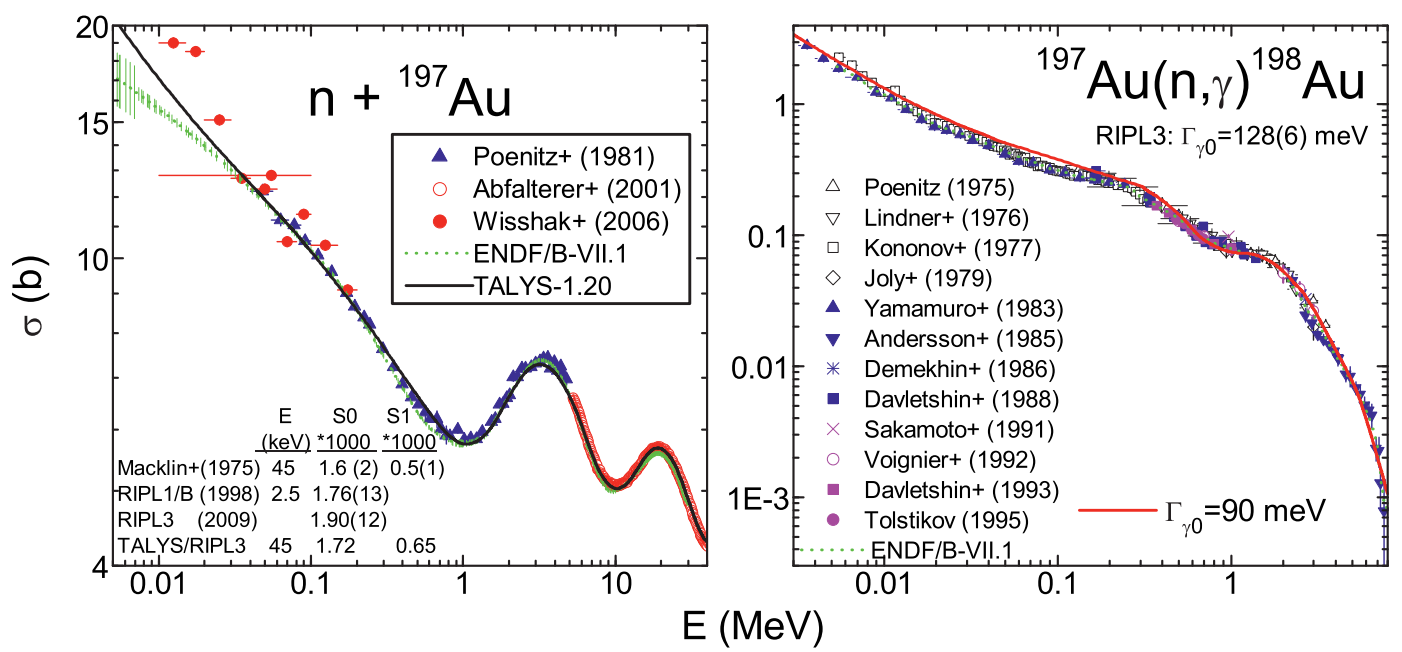

Fig. 1. (Color online) Comparison of measured [18], evaluated and calculated neutron total cross sections for ${ }^{197} \mathrm{Au}$ nucleus, by using the local neutron optical potential of Koning and Delaroche [16] (left), and $(n, \gamma)$ reaction cross sections on ${ }^{197} \mathrm{Au}$ by using the $\gamma$-ray strength functions corresponding to an average radiative width $\Gamma_{\gamma 0}$, of the s-wave neutron resonances, of $\sim 90 \mathrm{meV}$ (right).

\subsection{The $\gamma$-ray strength functions}

The modified energy-dependent Breit-Wigner (EDBW) model [24,25] was used for the electric dipole $\gamma$-ray strength functions $f_{E 1}\left(E_{\gamma}\right)$ of main importance for calculation of the $\gamma$-ray transmission coefficients. The systematic EDBW-model correction factor $\mathrm{F}_{S R}$ has been chosen in order to provide $f_{E 1}\left(E_{\gamma}\right)$ values close to the related experimental data and former calculations (Refs. [26,27]). At the same time we used the $\gamma$-ray strength function $f_{M 1}$ parameters of Ref. [27] as well as global estimations [28] of the $\gamma$-ray strength functions for the other multipoles $\lambda \leq 3$. The corresponding strength functions have finally been checked within the calculations of capture cross sections of ${ }^{197}$ Au nucleus in the neutron energy range from $\mathrm{keV}$ to $\sim 8 \mathrm{MeV}$, by using the OMP and nuclear level density parameters described below. The good agreement that we have obtained with the measured $(n, \gamma)$ reaction cross sections (Fig. 1) provides thus the validation of the adopted $\gamma$-ray strength functions in spite of the fact that at the same time an average radiative width $\Gamma_{\gamma 0}^{e x p}$ lower than the experimental [19] value resulted as it is also noted in Fig. 1.

\subsection{Nuclear level density}

The nuclear level densities were derived on the basis of the back-shifted Fermi gas (BSFG) formula [29], for the excitation energies below the neutron-binding energy, with the parameters $a$ and $\Delta$ [5] obtained by a fit of more recent experimental low-lying discrete levels [30] and $s$-wave nucleon resonance spacings $D_{0}$ [19]. Above the neutron binding we took into account the washing out of shell effects within the approach of Ignatyuk et al. [31] and Junghans et al. [32], and using the method of Koning and Chadwick [33] for fixing the appropriate shell correction energy. A transition range from the BSFG formula description to the higher energy approach has been chosen between the neutron binding energy and the excitation energy of $15 \mathrm{MeV}$, mainly in order to have a smooth connection. Concerning the level density spin distribution, we used firstly a variable ratio $I / I_{r}$ of the nuclear moment of inertia to its rigid-body value, between 0.5 for ground states, 0.75 at the neutron binding energy, and 1 around the excitation energy of $15 \mathrm{MeV}$. The results to be shown in the next section proved however the need of consideration of also a constant ratio $I / I_{r}$, equal with either 1 or 0.5 . Therefore we did the fit of low-lying discrete levels [30] and $s$-wave nucleon resonance spacings $D_{0}$ 


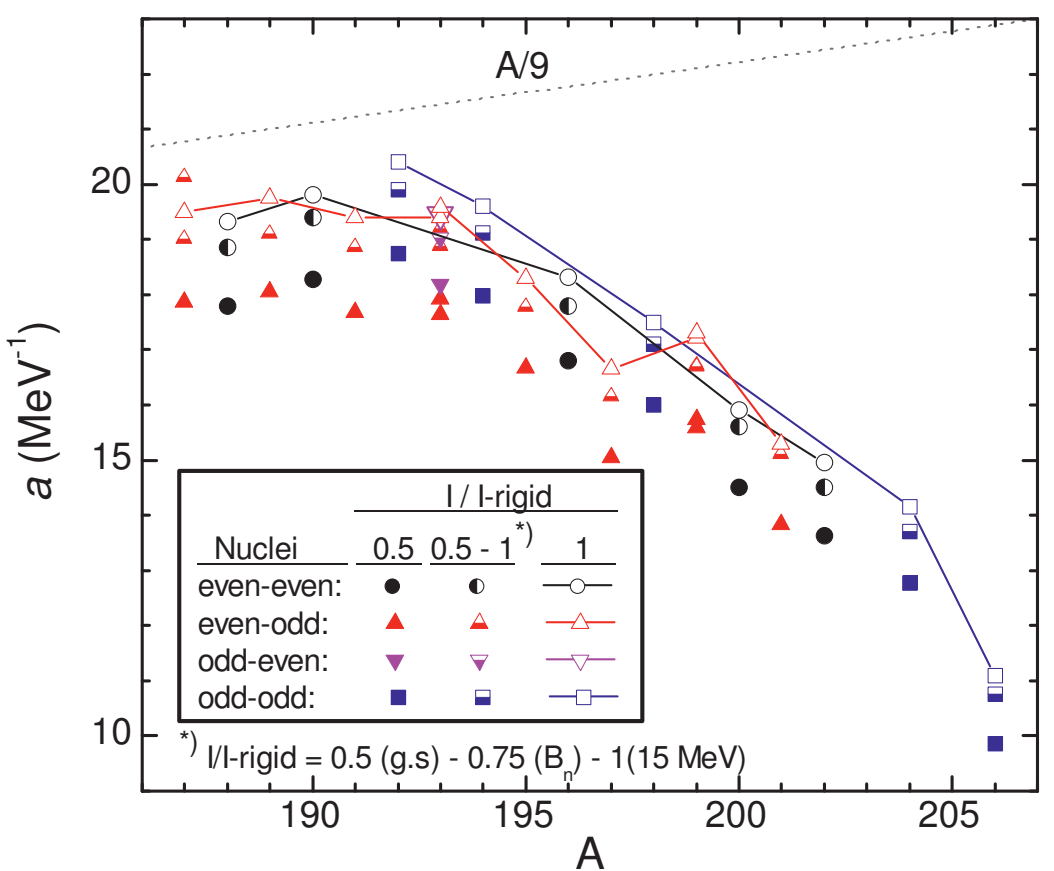

Fig. 2. (Color online) The BSFG-model nuclear level density parameter $a$ values obtained by fit of recent experimental low-lying discrete levels [30] and $s$-wave nucleon resonance spacings $D_{0}$ [19], corresponding to a variable ratio $I / I_{r}$ of the nuclear moment of inertia to its rigid-body value, between 0.5 for ground states and 0.75 at the neutron binding energy (half-solid symbols), and constant ratio $I / I_{r}$ equal with either 0.5 (solid symbols) or 1 (full symbols). The lines in the latest case just to guide the eye.

also for these constant $I / I_{r}$ values in the range $187<A<206$, the three sets of the $a$-parameter values being shown in Fig. 2. For the nuclei without resonance data we applied the smooth-curve method [28] by using average $a$-parameters, given by the narrow $A$-range systematics, for the fit of the low-lying discrete levels.

Concerning the particle-hole state density which for the PE description plays the same role as the nuclear-level density for statistical model calculations, a composite formula [15] was used within the GDH model with no free parameters except for the $\alpha$-particle state density $g_{\alpha}=A / 10.36 \mathrm{MeV}^{-1}$ [13].

\section{Results and discussion}

\subsection{The $(n, x n)$ reactions}

The agreement of the calculated cross sections with the measured data for the ${ }^{197} \mathrm{Au}(n, 2 n){ }^{196} \mathrm{Au}$ reaction is suitable especially with reference to the most recent and accurate experiment [2] as well as the ones within the last decade [35]. However it could be noted the larger spreading of data around the incident energy of $14 \mathrm{MeV}$ (Fig. 3, left), while our calculated values match the lower limit of the most recent data [35] in this energy range. Thus additional measurements would be helpful also here for real validation of the model calculations, otherwise one might consider any calculated results within even $\sim 30 \%$ in agreement with the available data. It is the same case for the evaluated data [34] from $\sim 14$ to $30 \mathrm{MeV}$, while however our calculations are well describing the data including the most recent ones. On the other hand, the too large error bars of the only several data measured above $30 \mathrm{MeV}$ make questionable a support of the model calculations. In any case the sensitivity of these calculations to the option adopted for the nuclear moment of inertia is so low that no conclusion is possible. 
$\mathrm{CNR} * 11$
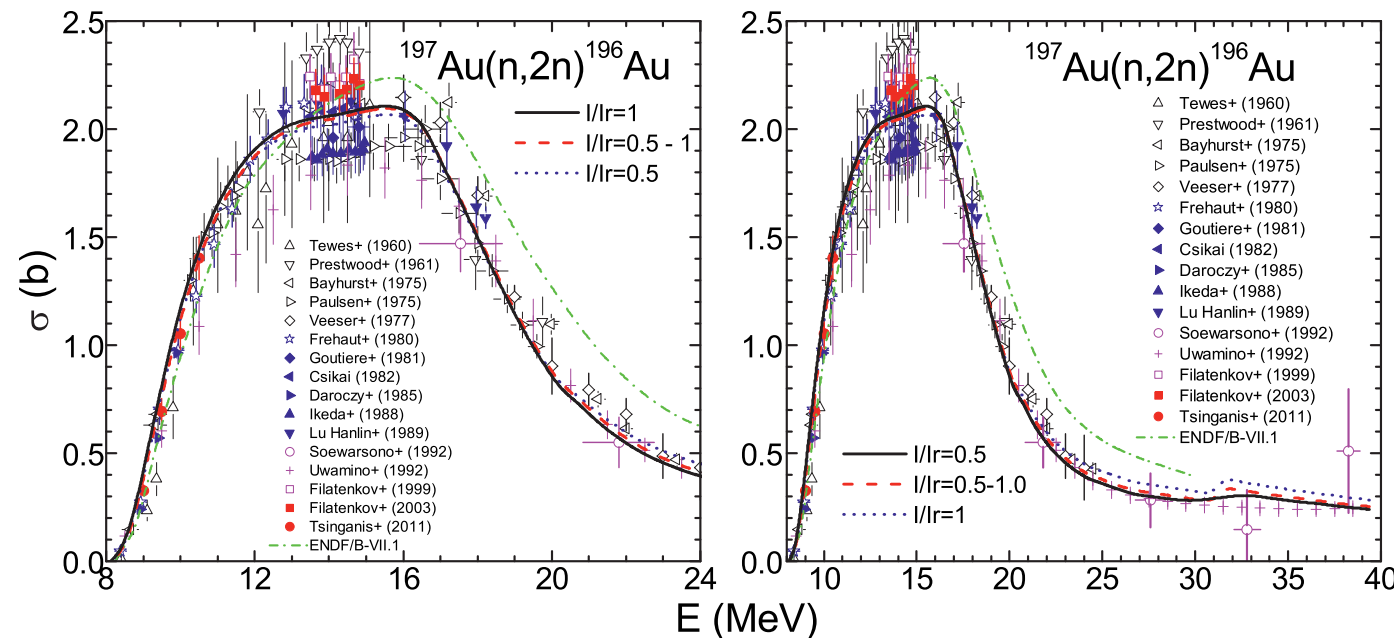

Fig. 3. (Color online) Comparison of experimental [18], ENDF/B-VII.1 evaluated [34], and calculated ${ }^{197} \mathrm{Au}(n, 2 n){ }^{196} \mathrm{Au}$ reaction cross sections by using the excitation-energy variable ratio $I / I_{r}$ of the nuclear moment of inertia to its rigid-body value (dash-dotted curve), or the constant ratios 0.5 (dotted) and 1 (solid), for incident energies up to $24 \mathrm{MeV}$ (left) and $40 \mathrm{MeV}$ (right).

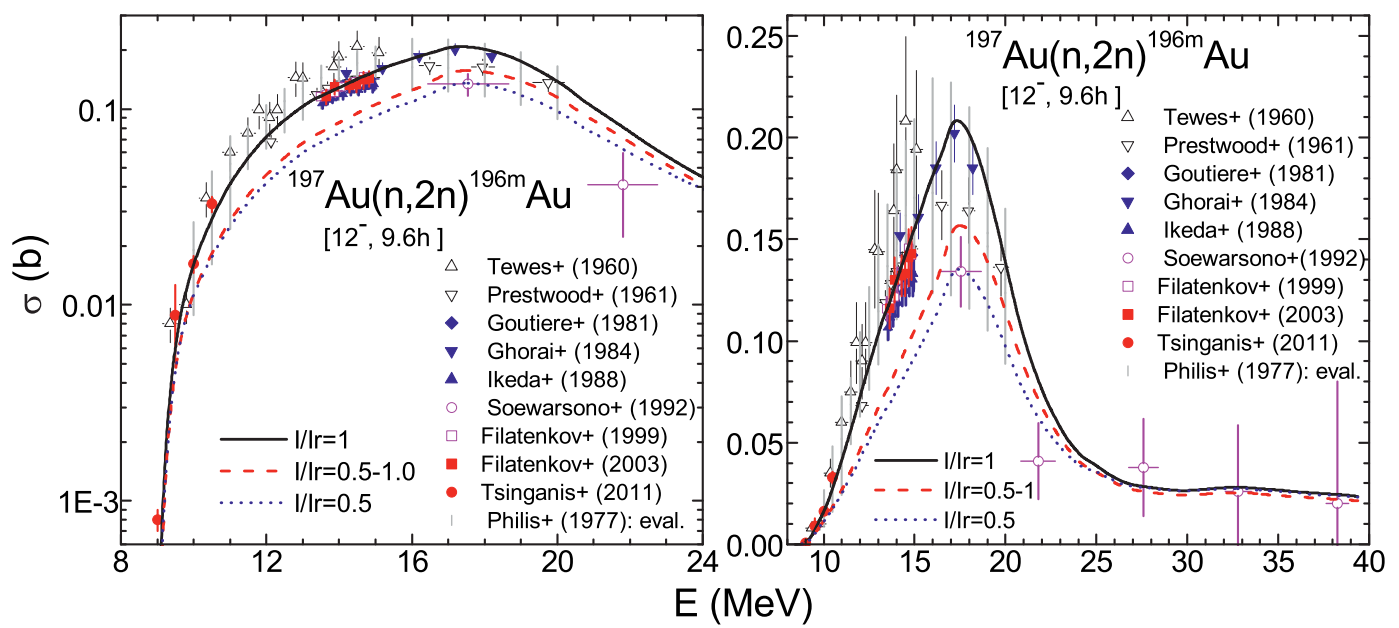

Fig. 4. (Color online) As for Fig. 3 (right), but for the ${ }^{197} \mathrm{Au}(n, 2 n){ }^{196} \mathrm{Au}^{m 2}$ reaction, with the corresponding spin, parity and lifetime shown between square brackets.

The comparison of the calculated and experimental cross sections for the population of the highspin second isomeric state also through the $(n, 2 n)$ reaction is a quite different case. This isomeric state is the 55th excited state of the ${ }^{196} \mathrm{Au}$ residual nucleus, at the top of the discrete levels taken into account in the SM calculations. Thus its population comes from the side feeding and continuum decay, so that it is fully determined by the nuclear level density and $\gamma$-ray strength functions. While the latter quantities were proved in the above Sec. 2.2 to be suitably considered, the model sensitivity to the nuclear moment of inertia assumption results to be so large (Fig. 4, left) that makes possible together of high accuracy of the recent measured data $[2,35]$ a certain conclusion on the real ratio $I / I_{r}$. Its value has thus resulted to be around 1, the other both involved options being definitely invalidated. 


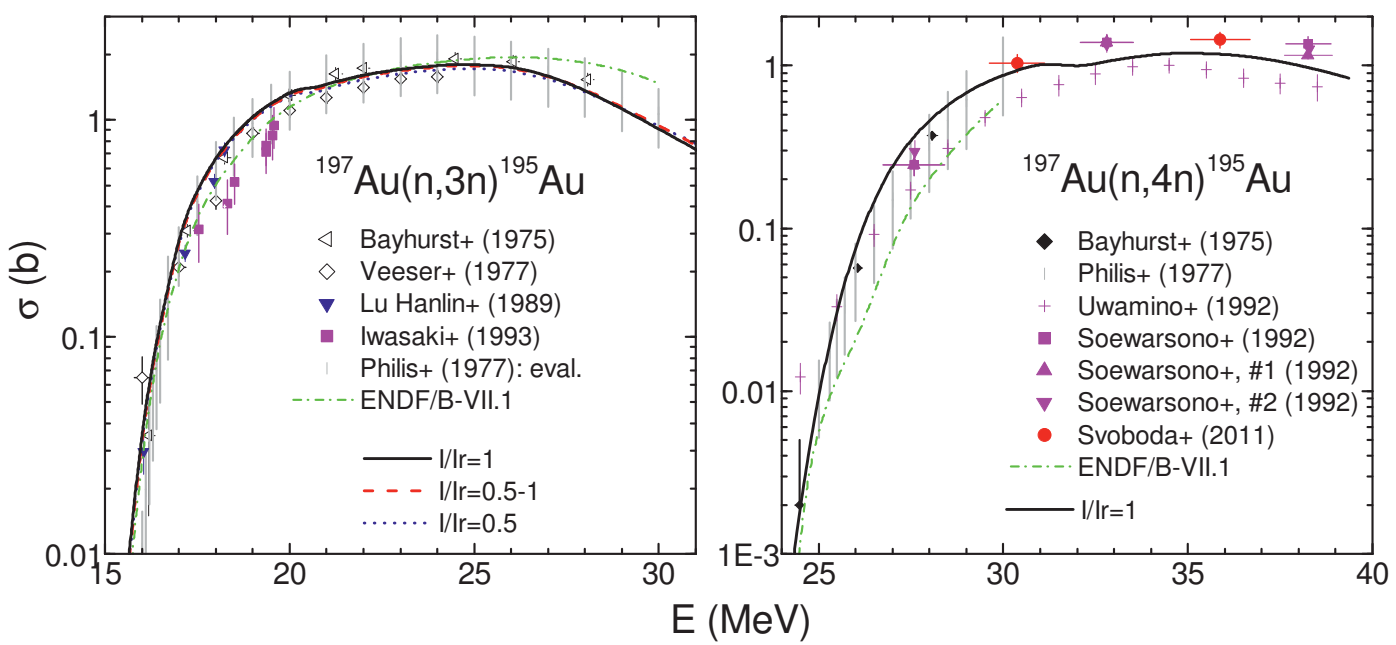

Fig. 5. Color online) As for Fig. 3 (right), but for the $(n, 3 n)$ reaction (left), and $(n, 4 n)$ reaction on ${ }^{197} \mathrm{Au}$ for the only case of a constant ratio $I / I_{r}=1$ (right).

Unfortunately even the more recent but only one data set available above $20 \mathrm{MeV}$ (Fig. 4, right) has so large error bars $(\geq 50 \%)$ that no further assessment can be concluded on either the correct nuclear moment of inertia or the key model quantities of the PE which becomes dominant at these energies (e.g., Refs. [36,37].

Similar cases of missing data with higher accuracy or better incident energy resolution are shown in Fig. 5 for the $(n, 3 n)$ and $(n, 4 n)$ reactions on the ${ }^{197} \mathrm{Au}$ nucleus. One may remark that, on the basis of the actual measured data and model calculations, it seems that the evaluated data accuracy remains rather unchanged within latest 30 years. On the other hand it is obvious the need of improved experimental data, in order to establish, e.g., the correctness of the change around the incident energy of 31 $\mathrm{MeV}$ of the $(n, 4 n)$ reaction excitation function. This change follows the start of PE contribution due to a higher angular momentum (e.g., Ref. [38]), and its positions depends by some PE assumptions within the GDH model as the Fermi energy value. Further data with increased accuracy and energy resolution may definitely contribute to the increase of the predictability power of actual phenomenological models while related microscopic models of a similar strength are presently not available.

\subsection{The $(n, p)$ reaction}

The unitary character of a reaction model analysis means a similar description of all reaction channels for which measured data exist, i.e. the $(n, p)$ and $(n, \alpha)$ reactions for the ${ }^{197} \mathrm{Au}$ target nucleus. Moreover, these reactions populate also well-known isomeric states, so that their study is a challenge concerning the present conclusions on the nuclear moment of inertia as well.

As regards the $(n, p)$ reaction, our calculation results describe rather well the most recent experimental data [35] for the population of the ground and isomeric states of the residual nucleus ${ }^{197} \mathrm{Pt}$ (Fig. 6), the similar case being obviously for the related isomeric ratio. However the sensitivity of the ${ }^{197} \mathrm{Au}(n, p){ }^{197} \mathrm{Pt}^{m}$ reaction cross sections is much lower than within previous case, obviously due to the lower isomeric state spin and excitation energy, as well as different decay scheme. Actually this comparison is just pointing out the particular case of the high-spin second isomeric state of the ${ }^{196} \mathrm{Au}$ nucleus. 


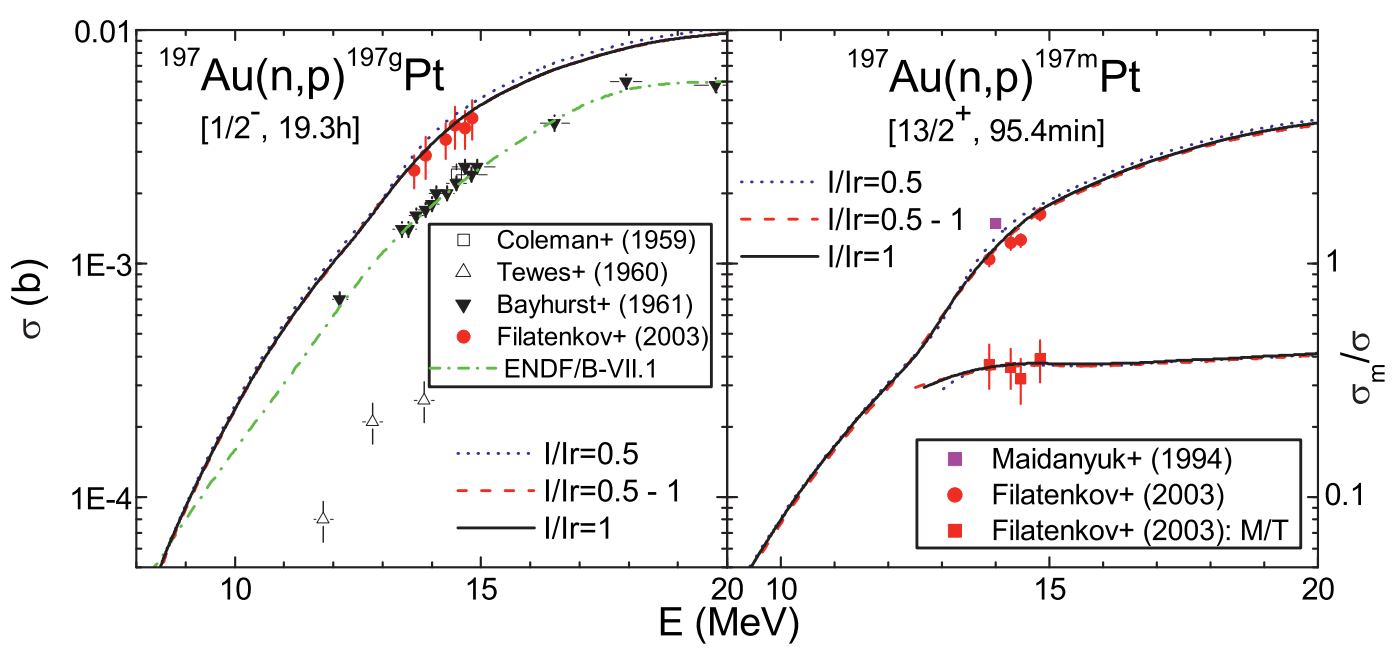

Fig. 6. (Color online) Comparison of experimental [18], ENDF/B-VII.1 evaluated [34], and calculated ( $n, p)$ reaction cross sections for the target nucleus ${ }^{197} \mathrm{Au}$ and population of the ground (left) and isomeric (right) states of the residual nucleus ${ }^{197} \mathrm{Pt}$ by using the same options for the ratio $I / I_{r}$ as for Figs. 3-5. The same comparison is shown for the isomeric ratio (right).

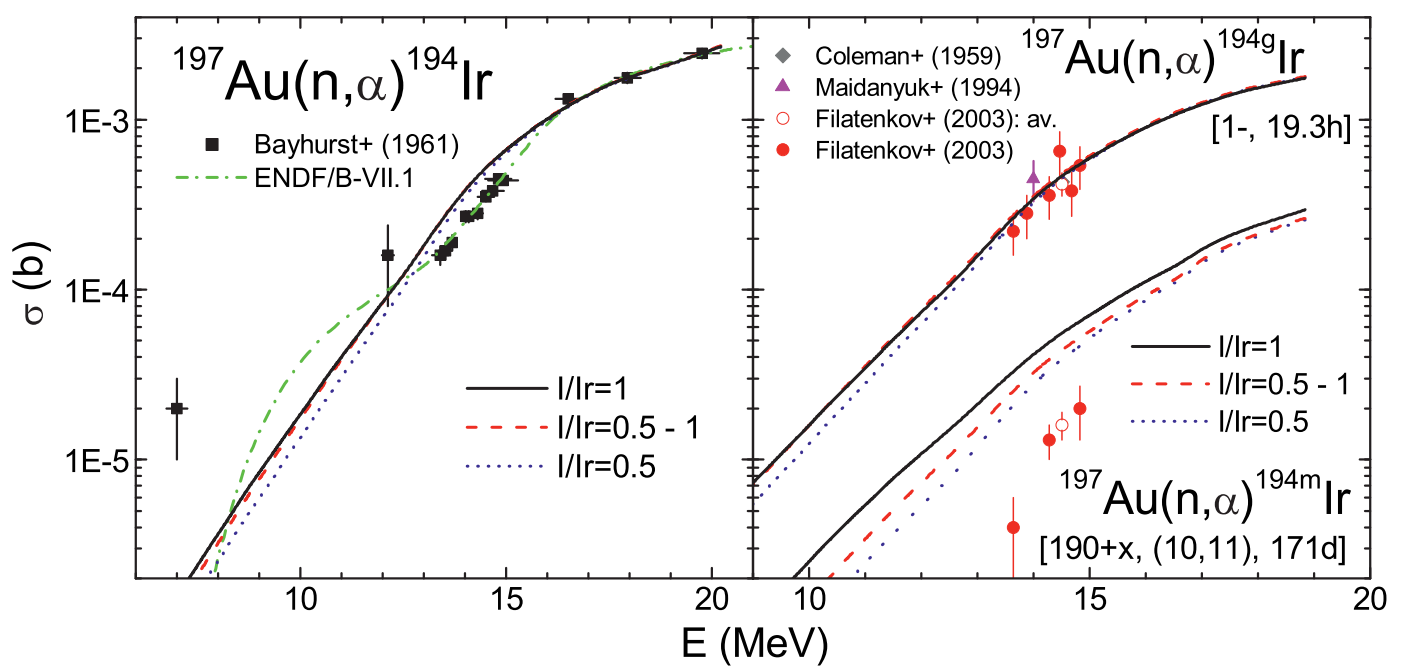

Fig. 7. (Color online) Comparison of experimental [18], ENDF/B-VII.1 evaluated [34], and calculated ( $n, \alpha)$ reaction cross sections for the target nucleus ${ }^{197} \mathrm{Au}$ (left), and population of the ground and isomeric states of the residual nucleus ${ }^{194} \operatorname{Ir}$ (right), by using the same options for the ratio $I / I_{r}$ as for Figs. 3-6.

\subsection{The $(n, \alpha)$ reaction}

While there is only one former measured data set of the total $(n, \alpha)$ reaction cross sections up to 20 $\mathrm{MeV}$, our calculated results are in agreement with it above $16 \mathrm{MeV}$ as well as around $12 \mathrm{MeV}$ but overestimate somehow the data around $14 \mathrm{MeV}$ (Fig. 7, left). However, in the latest energy range there are recent data [35] for the population of the ground state, that are well reproduced by our calculation. The unexpected issue has been, on the other hand, the large overestimation of the isomeric cross sections measured at the same time with those for the ground state population (Fig. 7, right). Actually this isomeric state has an uncertain excitation energy, between 190 and $440 \mathrm{keV}$, as well as 


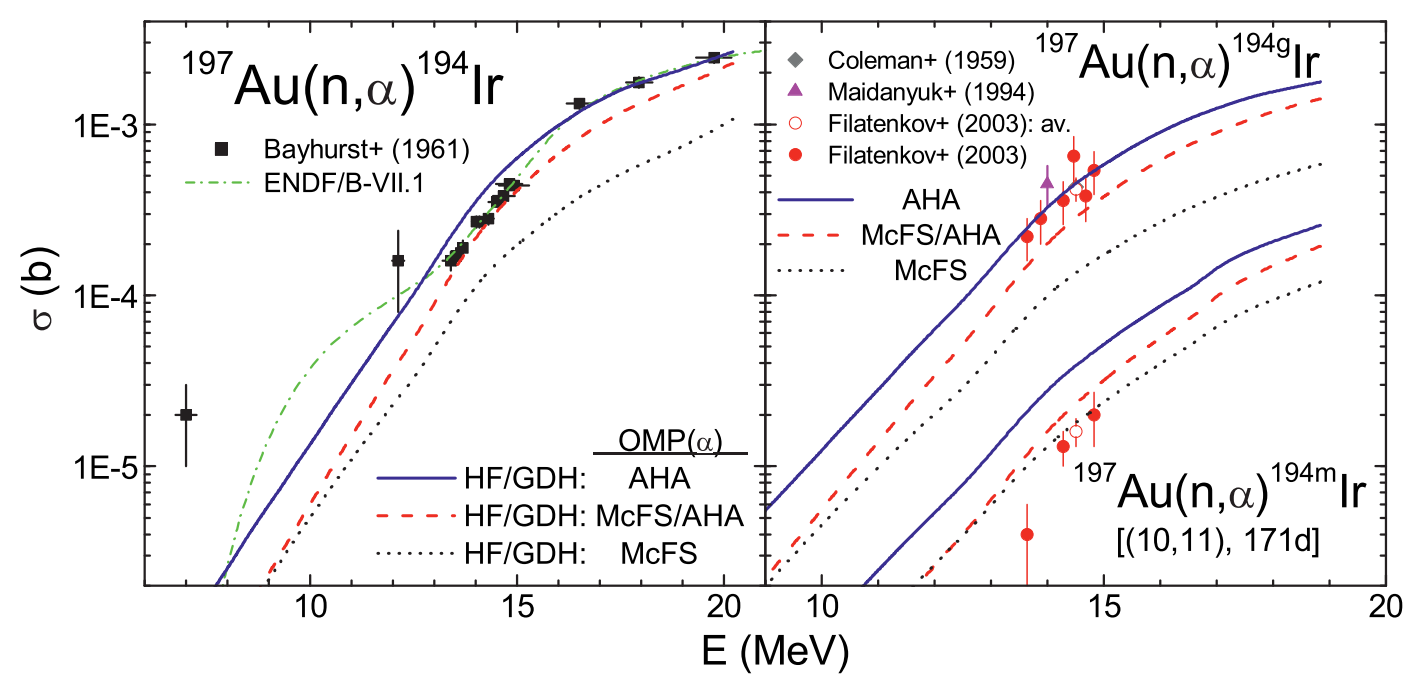

Fig. 8. (Color online) As for Fig. 7, but for reaction cross section calculations using, for the ratio $I / I_{r}=0.5$, the $\alpha$-particle OMP parameters of Ref. [22] either in both GDH (PE) model and SM model calculations (solid curves) or only in the former while in the latter are used the OMP parameters of McFadden and Satchler (dashed), as well as for the case of using the second OMP in both model calculation (dotted).

spin (either 10 or $11 \hbar$ ) and parity [39]. While the calculated isomeric cross sections are not sensitive to the excitation energy, assumed in these condition to be $440 \mathrm{keV}$ for our calculations, the spin value is quite important. We have considered, in agreement with the neighboring similar isotopes, the spin and parity $11^{-}$. This assumption leads obviously to lower reactions cross sections, the calculated results being still larger. The sensitivity of this calculated isomeric cross sections with respect to the option on the nuclear moment of inertia is in this case similar to the experimental errors, and the results that are closer to the measured data correspond to the ratio $I / I_{r}=0.5$.

On the other hand, there are still some significant questions marks on calculation of this reaction cross sections. One of them concerns the $\alpha$-particle OMP taken into account within both the SM and PE models. The effects of replacing the OMP of Ref. [22], established especially for the case of $\alpha$ particle emission, with that widely-used of McFadden and Satchler [40] are shown in Fig. 8 for the case of the ratio $I / I_{r}=0.5$. Firstly the replacement has been done only within the SM calculations, namely for the $\alpha$-particle transmission coefficients. Next, the imaginary OMP parameters of Ref. [40] replaced the former parameters [22] within the GDH calculation of the intra-nuclear transition rates. The corresponding effects are cumulative and both of them lead to the significant decrease of the reaction cross sections for population of the ground and isomeric states as well. Since the question of different OMP parameters in the incident and outgoing channels (e.g., Ref. [23]) should be firstly made clear, following the rather global consistent description of $\alpha$-particle OMP in the incident channel [41], for the time being we remain with the present inference of the half rigid-body value for the nuclear moment of inertia of the ${ }^{194}$ Ir nucleus obtained by $(n, \alpha)$ reaction. A comparison with the exact corresponding value $0.31 \pm 2$ [3] is not yet possible since there are not known the isomeric state spin and the $\alpha$-particle OMP used in Ref. [3].

\section{Conclusions}

Following the request of the ITER, IFMIF and DEMO nuclear engineering design for improvement of nuclear models and update of databases, questions of consistent model analysis of all available fast-neutron reaction data for the ${ }^{197} \mathrm{Au}$ target nucleus have been discussed. It becomes thus possible to describe well most of these data and to obtain a definite proof on the one rigid body value for the 


\section{$\mathrm{CNR} * 11$}

nuclear moment of inertia of the ${ }^{196} \mathrm{Au}$ nucleus, by the analysis of its high-spin second isomeric state population through the $(n, 2 n)$ reaction. A still open question is related however to the inference of the half rigid-body value for the nuclear moment of inertia of the ${ }^{194}$ Ir nucleus obtained by $(n, \alpha)$ reaction.

On the other hand, since the corresponding model assumptions are better proved by analysis of the data above 20-30 MeV, where only several data with large uncertainties are known, it has resulted the usefulness of further measurements to be performed at large-scale facilities as SPIRAL-2 and n_TOF, for incident energies up to 40 as well as $100 \mathrm{MeV}$. The new data to be thus obtained will answer the demands of the fusion technology programmes and will make also possible suitable validation of nuclear models in the energy range between the low and intermediate energies. The feedback of the improved nuclear model calculations at these energies will be direct on providing with an appropriate accuracy the additional data requested by these applications. An obvious case in this respect, also concerned in this work, is the knowledge of the $\alpha$-particle emission in fast-neutron induced reactions that is quite important for radiation damage assessment. However there are question marks on both the $\alpha$-particle OMP in the emergent channel and the pre-equilibrium emission of $\alpha$-particles. Additional measurements of $(n, \alpha)$ reaction cross sections at least up to $40-50 \mathrm{MeV}$ will be thus of equal importance for basic research and fusion technology.

\section{Acknowledgments}

This work was partly supported by the National Authority for Scientific Research under the project No. PN-09-37-01-05.

\section{References}

1. N. Patronis, C. T. Papadopoulos, S. Galanopoulos, M. Kokkoris, G. Perdikakis, R. Vlastou, A. Lagoyannis and S. Harissopulos, Phys. Rev. C 75, 034607 (2007).

2. A. Tsinganis, M. Diakaki, M. Kokkoris, A. Lagoyannis, E. Mara, C. T. Papadopoulos, and R. Vlastou, Phys. Rev. C 83, 024609 (2011).

3. M. S. Uddin, S. Sudár, and S. M. Qaim, Phys. Rev. C 84, 024605 (2011).

4. V. Semkova, V. Avrigeanu, T. Glodariu, A. J. Koning, A.J.M. Plompen, D.L. Smith, S. Sudar, Nucl. Phys. A 730, 255 (2004).

5. V. Avrigeanu, T. Glodariu, A. J. M. Plompen, and H. Weigmann, J. Nucl. Sci. Technol. Suppl. 2, 746 (2002).

6. X. Ledoux et al., in 11th Int. Conf. on Applications of Nuclear Techniques Crete, Greece, June 12-18, 2011 (in press); http://pro.ganil-spiral2.eu/spiral2/instrumentation/nfs .

7. E. Chiaveri et al. (The n_TOF Collaboration), in: Third International Workshop on Compound Nuclear Reactions and Related Topics, Sept. 19-23, 2011, Prague, EPJ Web of Conferences (in press); https://twiki.cern.ch/twiki/bin/view/NTOF/ .

8. M. Avrigeanu, V. Avrigeanu, STAPRE-H95 Computer Code, IPNE Report NP-86-1995, Bucharest, 1995 and references therein; News NEA Data Bank 17, 22 (1995).

9. M. Blann, H.K. Vonach, Phys. Rev. C 28, 1475 (1983).

10. M. Blann, Nucl. Phys. A 213, 570 (1973).

11. M. Uhl, B. Strohmaier, Report IRK-76/01, IRK, Vienna, 1981.

12. M. Avrigeanu, M. Ivascu, V. Avrigeanu, Z. Phys. A 329, 177 (1988) ; 335 (1990) 299.

13. E. Gadioli, E. Gadioli-Erba, Z. Phys. A 299, 1 (1981).

14. M. Avrigeanu, V. Avrigeanu, J. Phys. G: Nucl. Part. Phys. 20, 613 (1994).

15. M. Avrigeanu, V. Avrigeanu, Comp. Phys. Comm. 112, (1998); A.Harangozo, I.Stetcu, M.Avrigeanu, V.Avrigeanu, Phys. Rev. C 58, (1998).

16. A. J. Koning and J. P. Delaroche, Nucl. Phys. A713, 231 (2003).

17. J.P. Delaroche, Ch. Lagrange, J. Salvy, IAEA-190 (IAEA, Vienna, 1976), vol. 1, p. 251.

18. Experimental Nuclear Reaction Data (EXFOR), www-nds.iaea.or.at/exfor.

19. R. Capote et al., Nucl. Data Sheets 110, 3107(2009); http://www-nds.iaea.org/RIPL-3/ . 
20. K. Wisshak, F. Voss, F. K"appeler, M. Krtička, S. Raman, A. Mengoni, and R. Gallino, Phys. Rev. C 73, 015802 (2006).

21. A. J. Koning, S. Hilaire, and M. C. Duijvestijn, TALYS-1.0, in: Proc. Int. Conf. on Nuclear Data for Science and Technology, Nice, 2007, edited by O. Bersillon et al. (EDP Sciences, Paris, 2008), p. 1003., p. 211; version TALYS-1.2, December 2009; http://www.talys.eu/home/.

22. V. Avrigeanu, P.E. Hodgson, and M. Avrigeanu, Phys. Rev. C 49, 2136 (1994).

23. M. Avrigeanu, W. von Oertzen, and V. Avrigeanu, Nucl. Phys. A 764, 246 (2006).

24. D. G. Gardner and F. S. Dietrich, Report UCRL-82998, LLNL-Livermore, 1979.

25. M. Avrigeanu, V. Avrigeanu, G. Cata, and M. Ivascu, Rev. Roum. Phys. 32, 837 (1987).

26. S. Joly, Nucl. Sci. Eng. 94, 94 (1986).

27. J. Kopecky and M. Uhl, Phys. Rev. C 41, 1941 (1990).

28. C. H. Johnson, Phys. Rev. C 16, 2238 (1977).

29. H. Vonach, M. Uhl, B. Strohmaier, B.W. Smith, E.G. Bilpuch, and G. E. Mitchell, Phys. Rev. C 38, 2541 (1988).

30. Evaluated Nuclear Structure Data File (ENSDF), http://www.nndc.bnl.gov/ensdf/index.jsp .

31. A. V. Ignatyuk, G. N. Smirenkin, and A. S. Tishin, Yad. Fiz. 21 (1975) 485; Sov. J. Nucl. Phys. 21, 255 (1976).

32. A. R. Junghans, M. de Jong, H.-G. Clerc, A. V. Ignatyuk, G.A. Kudyaev, and K.-H. Schmidt, Nucl. Phys. A 629, 635 (1998).

33. A. J. Koning and M. B. Chadwick, Phys. Rev. C 56, 970 (1997).

34. M. B. Chadwick, P. Obložinský, M. Herman, N. M. Greene, R. D. McKnight, D. L. Smith et al., Nucl. Data Sheets 107, 2931 (2006).

35. A. A. Filatenkov and S. V. Chuvaev, Khlopin Radium Institute Preprint KRI-259, CNIIatominform, Moscow, 2003.

36. V. Avrigeanu, R. Eichin, R.A. Forrest, H. Freiesleben, M. Herman, A.J. Koning, K. Seidel, Nucl. Phys. A765, 1 (2006).

37. M. Avrigeanu, S. Chuvaev, A. A. Filatenkov, R. A. Forrest, M. Herman, A. J. Koning, A. J. M. Plompen, F. L. Roman, and V. Avrigeanu, Nucl. Phys. A806, 15 (2008).

38. P. Reimer, V. Avrigeanu, A.J.M. Plompen, and S.M. Qaim, Phys. Rev. C 65, 014604 (2001).

39. Balraj Singh, Nucl. Data Sheets 107, 1531 (2006).

40. L. McFadden and G.R. Satchler, Nucl. Phys. A 84, 177 (1966).

41. M. Avrigeanu, A. C. Obreja, F. L. Roman, V. Avrigeanu, and W. von Oertzen, At. Data Nucl. Data Tables 95, 501 (2009); M. Avrigeanu and V. Avrigeanu, Phys. Rev. C 82, 014606 (2010). 